\title{
Comparación in vitro de la microfiltración apical del MTA ProRoot y Angelus en dientes monorradiculares
}

\section{In vitro comparison of the MTA microlakage ProRoot apical and Angelus in teeth single-rooted}

\author{
Romero Romero GE*, Ramos Manotas J**, Díaz Caballero A***
}

\section{RESUMEN}

Antecedentes: Uno de los retos en endodoncia es la obturación de los conductos con ápices abiertos permitiendo su sellado, evitando la microfiltración y la salida del material obturador hacia los tejidos periapicales, por esta razón actualmente se utilizan materiales como el Mineral Trióxido Agregado (MTA) para proporcionar un tope apical que garantice el sellado del conducto radicular evitando la contaminación bacteriana.

Objetivos: Comparar la microfiltración apical in vitro del MTA de dos casas comerciales con y sin hidróxido de calcio en dientes monorradiculares con ápices amplios.

Materiales y métodos: Para este estudio se utilizaron 80 dientes humanos monorradiculares extraídos, divididos en 4 grupos, MTA "Proroot" con y sin hidróxido de calcio y MTA "Angelus" con y sin hidróxido de calcio, sumergidos en una sustancia buffer simulando el periápice y posteriormente se realizó coloración con tinta china y evaluación de la microfiltración por medio del proceso de diafanación.

Resultados: En el estudio todos los grupos presentaron algún grado de microfiltración, siendo los de mayor frecuencia los dientes obturados con MTA Angelus sin hidróxido de calcio y el que menos presentó fueron los obturados con MTA Proroot sin hidróxido de calcio.

Conclusiones: Tanto el MTA Angelus como el MTA Proroot se pueden usar como barrera apical sin necesidad de usar hidróxido calcio, sin embargo es necesario afinar las técnicas y procedimientos en futuros estudios que permitan la toma de decisiones clínicas.

Palabras clave: Mineral trióxido agregado, filtración, hidróxido de calcio. (MeSH Database).

\section{SUMMARY}

Background: One of the challenges in endodontics is root canal filling with open and wide apex, which allowing it to seal, preventing microleakage and the output of the filling material into periapical tissues, therefore currently used materials such as Mineral trioxide aggregate (MTA) to provide an apical stop to ensure the sealing of the root canal to avoid bacterial contamination.

Objectives: To compare the in vitro apical microleakage of MTA in two trading houses with and without calcium hydroxide in teeth with apical single-rooted wide.

* Odontóloga, Pontificia Universidad Javeriana. Residente postgrado de endodoncia Universidad de Cartagena.

** Odontólogo Universidad de Cartagena. Magister en Endodoncia. Profesor Titular Facultad de Odontología Universidad de Cartagena. Docente Postgrado de Endodoncia Universidad de Cartagena

*** Odontólogo, Universidad de Cartagena. Especialista en Periodoncia, Pontificia Universidad Javeriana. Profesor titular Universidad de Cartagena. Director grupo de investigaciones GITOUC. 
Materials and methods: For this study 80 extracted human teeth were used single-rooted divided into 4 groups, MTA "ProRoot" with and without calcium hydroxide and MTA Angelus with and without calcium hydroxide, immersed in a buffer substance and simulating periapical subsequently made with India ink staining and evaluation of microleakage through diafanación process.

Results: In the study all groups showed some degree of microleakage, the most frequent being filled teeth with MTA Angelus without calcium hydroxide and the least presented were filled with MTA ProRoot without calcium hydroxide.

Conclusions: Both the MTA and the MTA Angelus ProRoot can be used as apical barrier without the use of calcium hydroxide, however it is necessary to refine the techniques and procedures in future studies to clinical decision making.

Key words: Mineral Trioxide Aggregate (MTA), leakage, calcium hydroxide. (MeSH Database).

Fecha de recepción: 10 de abril de 2011.

Aceptado para publicación: 3 de junio de 2011.

Romero Romero GE, Ramos Manotas J, Díaz Caballero A. Comparación in vitro de la microfiltración apical del MTA ProRoot y Angelus en dientes monorradiculare. Av. Odontoestomatol 2012; 28 (3): 125-131.

\section{INTRODUCCION}

Uno de los principales factores que llevan al fracaso de la endodoncia es la obturación incompleta del conducto radicular y la falta de sellado apical, por lo que constituye la mayor preocupación del odontólogo realizar una obturación hermética que asegure una prolongada y útil permanencia del diente tratado (1).

Desde hace años se estudian las propiedades de los materiales que se encuentran en el mercado esperando que cumplan con el mayor número de requisitos de un material ideal postulado por Grossman para favorecer el éxito del tratamiento. Uno de los mayores inconvenientes que presentan los materiales de obturación es la falta de sellado hermético favoreciendo la microfiltración de bacterias y endotoxinas a través del conducto, impidiendo la reparación periapical que conlleva a un fracaso del tratamiento endodóntico $(2,3)$.

Este sellado hermético se hace más difícil en dientes que presentan forámenes apicales muy amplios y/o irregulares como en los casos de reabsorción radicular, perforaciones apicales y dientes necróticos de ápices inmaduros, que tienen el riesgo de extruir el material de obturación hacia los tejidos periapicales, lo que lleva al odontólogo a buscar una forma de lograr un tope apical con la ayudada de materiales como el hidróxido de calcio y actualmente el Mineral Trióxido Agregado (MTA) (4-6).

El mineral trióxido agregado entró al mercado en 1993 y fue desarrollado por Torabinejad en 1990 en la Universidad de Loma Linda en California como un compuesto potencial que sella el camino de las comunicaciones entre el sistema de conductos radiculares y la superficie externa del diente. Se indica como alternativa al hidróxido de calcio, además se recomienda su uso como barrera apical, por su capacidad de sellado, de endurecer en presencia de sangre y su biocompatibilidad; mostró éxito a largo plazo cuando se utiliza como material de reparación de perforaciones, lo que podría deberse a su capacidad para inducir la formación de tejido duro. Por ser un material de fraguado entre 15 minutos y 3 horas, brinda la ventaja de obtener el tapón apical en una sola cita, reduciendo el tiempo de tratamiento entre citas (7-9).

Este material recibió la aceptación por la Food and Drug Administration (FDA) y se encuentra disponible en el comercio como MTA ProRoot (Dentsply Tulsa, USA) en la forma gris y blanca y el MTA Angelus (Soluciones Odontológicas, Londrina, Brasil) (7). 
La mayor ventaja del mineral trióxido agregado sobre otros materiales se debe a sus propiedades, entre ellas el tiempo de endurecimiento 15 minutos para el Angelus y 24 horas para el ProRoot; su Ph es alcalino (el MTA ProRoot tiene un ph de 12,51 y del MTA Angelus es 12,0$)$ lo que lo hace similar al del hidróxido de calcio, proporcionándole efectos antibacterianos; fuerza compresiva baja; insoluble en presencia de humedad, garantizando un excelente sellado marginal; tiene una radiopacidad superior a la de la dentina y del tejido óseo facilitando su visualización en los controles radiográficos; presenta baja citotoxicidad. Estudios de biocompatibilidad con osteoblastos sugieren que puede promover la cicatrización a través de la estimulación del metabolismo óseo (6, 7, 10-12).

El MTA es indicado en recubrimientos pulpares, pulpotomías y apicoformación. La creación de la barrera apical con MTA está indicada en dientes con pulpas necróticas y ápices abiertos. También es usado en perforaciones radiculares y obturaciones retrógradas en cirugía endodóntica $(4,8)$.

El hidróxido de calcio fue introducido en odontología por Hermann en 1924, utilizado originalmente para promover la regeneración pulpar cuando por alguna razón ésta se exponía al medio bucal. Posteriormente se encontraron otros usos como apexificador. Es uno de los fármacos utilizados como medicación intraconducto, usándose también en protección pulpar directa y pulpotomía vital. Posee excelentes propiedades como es su baja solubilidad en agua, posee un $\mathrm{Ph}$ muy alcalino, aproximadamente 12,4 , lo que le confiere un efecto altamente bactericida ya que las principales bacterias encontradas dentro de los conductos radiculares se desarrollan a un Ph entre 5 y 8,2 $(13,14)$.

La microfiltración apical se entiende como la penetración de fluidos, bacterias y sustancias químicas dentro del conducto radicular. La microfiltración en la interface del material de obturación y las paredes del conducto radicular, este espacio puede ser el producto de la deficiente adaptación del material de relleno a las paredes dentinarias, la solubilidad del material o la estabilidad dimensional del sellador. Se pueden dar dos interfaces potenciales de microfiltración entre la gutapercha y el sellador o entre el sellador y las paredes del conducto $(1,15)$.
Al sobrepasar el nivel crítico de microfiltración de un conducto endodónticamente tratado puede producirse una enfermedad periapical y/o alterar la reparación de los tejidos periapicales $(1,16)$.

El objetivo de este estudio fue comparar la microfiltración que se produce después de sellar el tercio apical con MTA ProRoot y MTA Angelus en raíces con ápices abiertos y si la colocación de una capa de hidróxido de calcio antes del MTA afecta su capacidad de sellado.

\section{MATERIALES Y METODOS}

Este estudio fue de tipo cuasi experimental comparativo, de intervención e in vitro, con control y manipulación de las variables y asignación aleatoria de los grupos, simulando los procedimientos clínicos en el laboratorio. Se utilizaron dientes humanos unirradiculares recién extraídos, con ápices cerrados, con ángulo de la curvatura radicular entre $10^{\circ}$ y $20^{\circ}$ y longitud radicular no mayor de $25 \mathrm{~mm}(17,18)$. La muestra consistió en 80 dientes que cumplieran con los criterios de inclusión. Luego fueron almacenados en suero fisiológico durante 24 horas antes de ser procesados para evitar su deshidratación (19). Se eliminaron las coronas a nivel de la unión amelocementaria con discos de diamante con motor de baja velocidad a 20.000 revoluciones por minuto (NSK). Se instrumentaron hasta el CDC con limas FlexoFile de primera y segunda serie (Denstply) llevando el diámetro apical hasta una lima 70 para simular conductos con ápices amplios e irregulares que dificultaran un excelente sellado apical, se irrigó entre lima y lima con hipoclorito de sodio al $5,25 \%$ (Figura 1) (20). Una vez terminada la instrumentación se colocó la raíz en un cubo de acrílico transparente de $2 \mathrm{~cm} \times 2 \mathrm{~cm}$ lleno de una sustancia que contenía un miligramo de agar en $1.000 \mathrm{~mm}$ de suero fisiológico con fosfato de buffer salino, compuesto por $8 \mathrm{mg}$ de cloruro de sodio, 0,3 $\mathrm{mg}$ de cloruro de potasio, 1,15 mg de fosfato disódico, 0,3 $\mathrm{mg}$ de fosfato de potasio y $0,3 \mathrm{mg}$ de azide de sodio; a un ph de 7,4 y 3,7 de temperatura (figura 2). Luego se fijaron los dientes desde su cuello en un orificio hecho en una de las caras del cubo y se sellaron herméticamente con una gota $(1 \mathrm{mg})$ de silicona sólida $(18,21)$. 


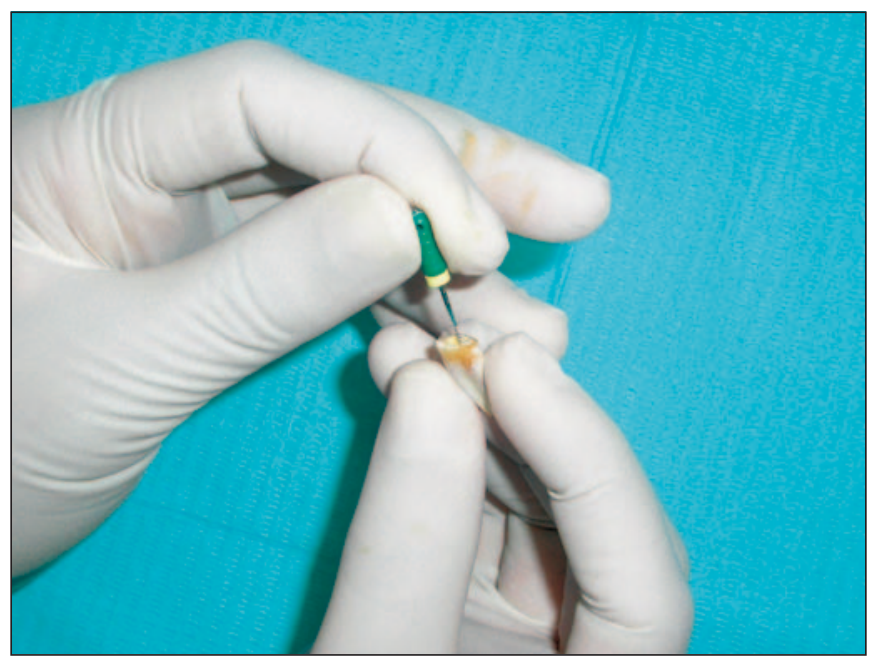

Fig. 1. Se observa instrumentación de los conductos hasta el CDC con limas FlexoFile de primera y segunda serie (Denstply) llevando el diámetro apical hasta una lima 70 para simular conductos con ápices amplios e irregulares.

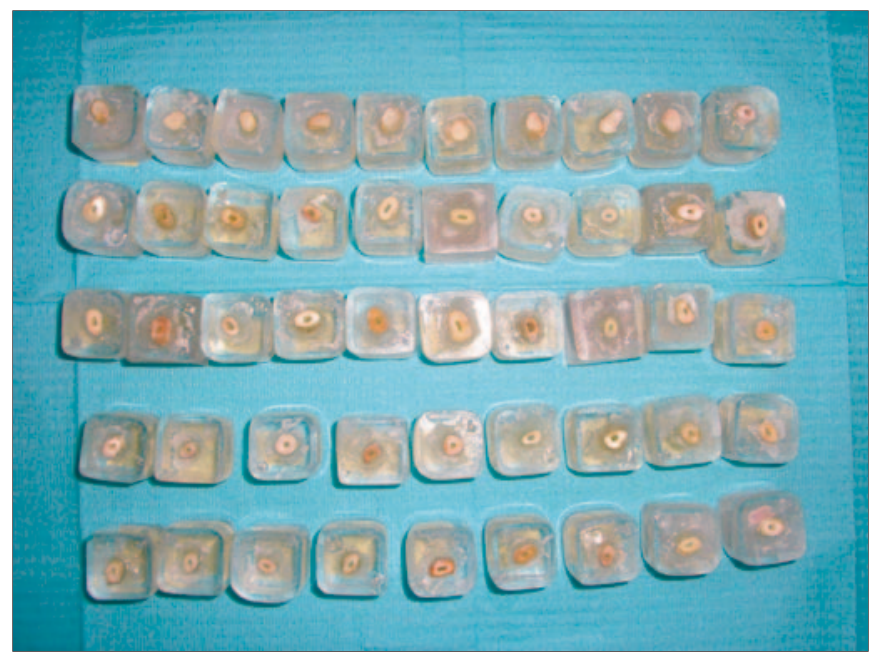

Fig. 2. Se observan las raíces dentro de los cubos de acrílico transparente de $2 \mathrm{~cm} \times 2 \mathrm{~cm}$ lleno de una sustancia que contenía un miligramo de agar en $1.000 \mathrm{~mm}$ de suero fisiológico con fosfato de buffer salino.

Las raíces se sumergieron en tinta china negra según el método Okumura-April (figura 3), esta se dividió en cuatro grupos de manera aleatoria cada uno con 20 dientes y se clasificaron de la siguiente manera: grupo A, 20 dientes obturados con hidróxido de calcio y MTA ProRoot; grupo B, 20 dientes obturados sin hidróxido de calcio y MTA ProRoot; grupo C, 20 dientes obturados con hidróxido de calcio y MTA Angelus y grupo D, 20 dientes obturados sin hidróxido de calcio y MTA Angelus.

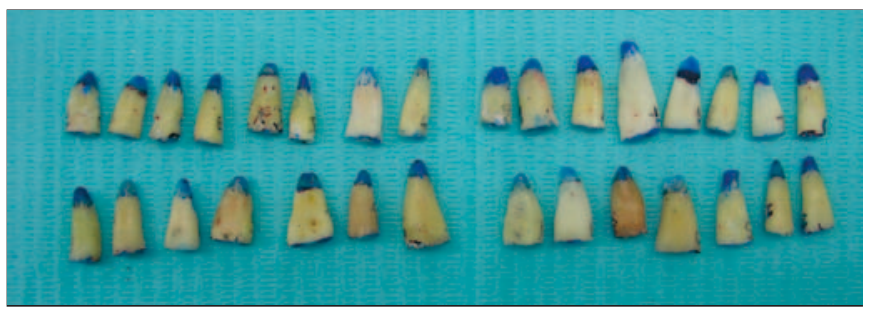

Fig. 3. Las raíces se sumergieron en tinta china negra según el método Okumura-Aprile.

Luego se realizó la obturación de los conductos con MTA hasta $3 \mathrm{~mm}$ de la abertura apical. A las 24 horas fueron sacados de la sustancia buffer, se secaron y los dos tercios superiores de la raíz se barnizaron con doble capa de esmalte de uñas excepto el tercio apical de la raíz mínimo hasta $3 \mathrm{~mm}$ del foramen apical (21).

Posteriormente las raíces se sumergieron en tinta china negra según el método Okumura-Aprile, figura 3. Las muestras fueron sometidas a un proceso de clarificación para hacer visible la filtración de tinta al conducto, siguiendo la técnica de Hosoya utilizando el kit de Eufar. Una vez transparentado los dientes, se procedió a medir la filtración apical con mediciones desde hasta $10 \mathrm{~mm}$ del ápice hacia cervical, se utilizó para esto una rejilla milimetrada 10/100XY montada en un microscopio de luz óptica marca Olympus SZ 60 con un aumento de 10/22 (22).

El análisis estadístico e interpretación de los datos se llevo a cabo mediante una prueba ANOVA y $t$ student, para determinar la cantidad de microfiltración y la frecuencia de microfiltración (23).

\section{RESULTADOS}

\section{Frecuencia de microfiltración}

El grupo de dientes que mayor frecuencia de microfiltración presentó fueron los tratados con MTA Angelus sin hidróxido de calcio con un porcentaje del $25 \%$, que corresponde al grupo D y el grupo de dientes que menor microfiltración presentó fueron los dientes tratados con MTA ProRoot sin hidróxido de calcio con un porcentaje de $10 \%$ que corresponden al grupo B (tabla 1, gráfico 1). 
TABLA 1.- FRECUENCIA DE MICROFILTRACIÓN ENTRE LOS CUATRO GRUPOS

\begin{tabular}{|l|c|c|c|}
\hline Grupos & $\begin{array}{c}\text { Número } \\
\text { de dientes }\end{array}$ & $\begin{array}{c}\text { Dientes con presencia } \\
\text { de microfiltracion }\end{array}$ & Porcentaje \\
\hline MTA (ProRoot)-CaOH & 20 & 4 & $20 \%$ \\
\hline MTA (ProRoot) & 20 & 2 & $10 \%$ \\
\hline MTA Angelus- CaOH & 20 & 3 & $15 \%$ \\
\hline MTA Angelus & 20 & 5 & $25 \%$ \\
\hline TOTAL & 80 & 14 & \\
\hline
\end{tabular}

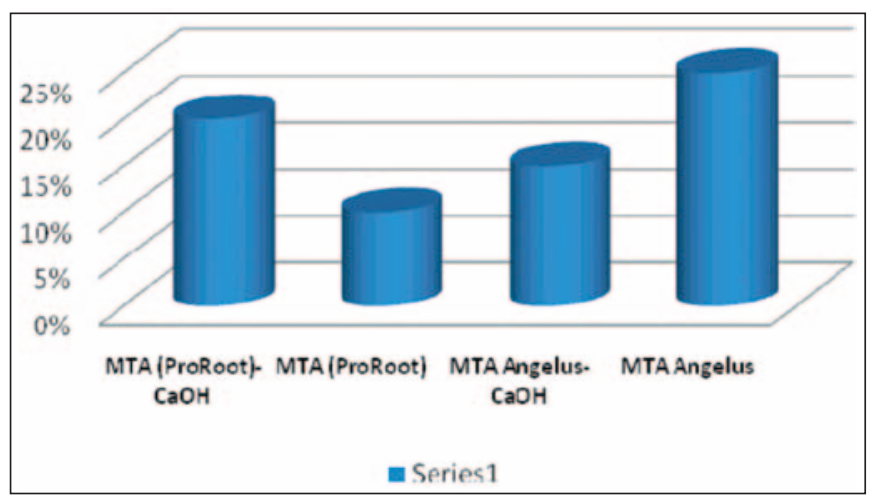

Gráfico 1. Muestra la comparación gráfica de la frecuencia de microfiltración de los cuatro grupos de estudio.

\section{Promedio de microfiltración}

El grupo de dientes que mayor cantidad de microfiltración presentó fueron los obturados con MTA Angelus sin hidróxido de calcio con un promedio de 1,51 con una desviación estándar (DS) de 2,81 y el grupo que menor cantidad de microfiltración presentó fueron los obturados con MTA ProRoot sin hidróxido de calcio con un promedio de 0,32 con una DS de 1,07. Al comparar los promedios de los cuatro grupos se observaron diferencias estadísticamente significativas. El grupo que mejor se comportó con respecto a la microfiltración fue el grupo B (tabla 2).

\section{DISCUSIÓN}

El presente estudio evaluó la microfiltración de dos marcas comerciales de MTA blanco (ProRoot y An-

\begin{tabular}{|l|l|}
\hline \multicolumn{2}{|c|}{ TABLA 2.- COMPARACIÓN DE } \\
PROMEDIOS DE MICROFILTRACIÓN \\
ENTRE LOS CUATRO GRUPOS
\end{tabular}

gelus) para determinar la eficacia que tiene como barrera apical en una sola cita, puesto que diversos estudios evaluaron la fórmula gris del MTA obteniendo buenos resultados clínicos. Además se quiso examinar si un apósito de hidróxido de calcio antes de la colocación apical del MTA afecta su adaptación y capacidad de sellado en condiciones in vitro experimentales $(6,24,25)$.

El apósito de hidróxido de calcio previo al MTA se utilizó principalmente para evitar la extrusión de éste hacia la región periapical. Sin embargo esto no se considera un problema clínico importante debido a que la biocompatibilidad del MTA está bien establecida. Según los resultados de este estudio, a nivel de todos los grupos solo se encontró un $17 \%$ de dientes 
con algún grado de microfiltración, siendo esta filtración distribuida en forma similar para los dos grupos obturados con y sin hidróxido de calcio. Según estos resultados no hubo diferencias estadísticas fuertes que puedan soportar el uso del MTA acompañado del uso del hidróxido de calcio, ya que se podría utilizar el MTA solo y los resultados serían similares. Esto podría reducir el tiempo de la técnica aplicada y una mejor comodidad para el paciente. Por otro lado en cuanto al uso de las dos marcas comerciales de MTA, no hay evidencia suficiente para descartar alguno de los dos, por lo tanto se debe seguir realizando investigaciones que logren aclarar este problema (26-28).

Se puede decir que el método de filtración con tinta china es apropiado ya que proporciona una medición cuantitativa de microfiltración de las muestras experimentales, teniendo en cuenta que el tamaño molecular de esta tinta es menor que el de las bacterias por lo tanto si se encuentra microfiltración con tinta, posiblemente filtra menos con bacterias (22).

\section{CONCLUSIÓN}

Dentro de las limitante actuales del estudio, se concluye que tanto el MTA Angelus como el MTA ProRoot se pueden usar como barrera apical sin necesidad de usar el hidróxido de calcio sin embargo es necesario afinar las técnicas y procedimientos en futuros estudios que permitan la toma de decisiones clínicas.

\section{AGRADECIMIENTOS}

A los residentes de endodoncia Anny González Ochoa, Ingrid Romero Herrera y Martha Turcios Baldovino por su colaboración en la recopilación de los datos experimentales

\section{BIBLIOGRAFÍA}

1. Fogel HM, Peikoff MD. Microleakage of root-end filling materials. J Endod. 2001 Jul;27(7):456-8.

2. Andelin WE, Browning DF, Hsu GH, Roland DD, Torabinejad M. Microleakage of resected MTA. J Endod. 2002 Aug;28(8):573-4.
3. Hosoya N, Nomura M, Yoshikubo A, Arai T, Nakamura J, Cox CF. Effect of canal drying methods on the apical seal. J Endod. 2000 May; 26(5):292-4.

4. Chng HK, Islam I, Yap AU, Tong YW, Koh ET. Properties of a new root-end filling material. J Endod. 2005 Sep;31(9):665-8.

5. Yildirim T, Tasdemir T, Orucoglu $H$. The evaluation of the influence of using MTA in teeth with post indication on the apical sealing ability. Oral Surg Oral Med Oral Pathol Oral Radiol Endod. 2009 Sep;108(3):471-4.

6. Menezes R, Bramante CM, Letra A, Carvalho VG, García RB. Histologic evaluation of pulpotomies in dog using two types of mineral trioxide aggregate and regular and white Portland cements as wound dressings. Oral Surg Oral Med Oral Pathol Oral Radiol Endod. 2004 Sep;98 (3):376-9.

7. Torabinejad M, Parirokh M. Mineral trioxide aggregate: a comprehensive literature review—part II: leakage and biocompatibility investigations. J Endod. Feb;36(2):190-202.

8. Parirokh $M$, Torabinejad $M$. Mineral trioxide aggregate: a comprehensive literature reviewPart III: Clinical applications, drawbacks, and mechanism of action. J Endod. Mar;36(3):40013.

9. Schmitt D, Lee J, Bogen G. Multifaceted use of ProRoot MTA root canal repair material. Pediatr Dent. 2001 Jul-Aug;23(4):326-30.

10. Song JS, Mante FK, Romanow WJ, Kim S. Chemical analysis of powder and set forms of Portland cement, gray ProRoot MTA, white ProRoot MTA, and gray MTA-Angelus. Oral Surg Oral Med Oral Pathol Oral Radiol Endod. 2006 Dec;102(6):809-15.

11. Saghiri MA, Lotfi M, Saghiri AM, Vosoughhosseini $\mathrm{S}$, Fatemi A, Shiezadeh V, et al. Effect of $\mathrm{pH}$ on sealing ability of white mineral trioxide aggregate as a root-end filling material. J Endod. 2008 Oct;34(10):1226-9. 
12. Camilleri J, Pitt Ford TR. Mineral trioxide aggregate: a review of the constituents and biological properties of the material. Int Endod J. 2006 Oct;39(10):747-54.

13. Gomes BP, Ferraz CC, Garrido FD, Rosalen PL, Zaia AA, Teixeira FB, et al. Microbial susceptibility to calcium hydroxide pastes and their vehicles. J Endod. 2002 Nov;28(11):758-61.

14. Shabahang S, Torabinejad M, Boyne PP, Abedi $\mathrm{H}$, McMillan P. A comparative study of root-end induction using osteogenic protein-1, calcium hydroxide, and mineral trioxide aggregate in dogs. J Endod. 1999 Jan;25(1):1-5.

15. Torabinejad M, Rastegar AF, Kettering JD, Pitt Ford TR. Bacterial leakage of mineral trioxide aggregate as a root-end filling material. J Endod. 1995 Mar;21(3):109-12.

16. Torabinejad M, Watson TF, Pitt Ford TR. Sealing ability of a mineral trioxide aggregate when used as a root end filling material. J Endod. 1993 Dec;19(12):591-5.

17. Zheng QH, Zhou XD, Jiang Y, Sun TQ, Liu CX, Xue $\mathrm{H}$, et al. Radiographic investigation of frequency and degree of canal curvatures in Chinese mandibular permanent incisors. J Endod. 2009 Feb;35(2):175-8.

18. Schneider SW. A comparison of canal preparations in straight and curved root canals. Oral Surg Oral Med Oral Pathol. 1971 Aug;32(2):271-5.

19. Ghersel EL, Guedes-Pinto AC, Ciamponi AL. [Effect of the storage method on microleakage of primary teeth restored with different bonding systems: in vitro study]. Pesqui Odontol Bras. 2001 Jan-Mar;15(1):29-34.

20. Kim US, Shin SJ, Chang SW, Yoo HM, Oh TS, Park DS. In vitro evaluation of bacterial leakage resistance of an ultrasonically placed mineral trioxide aggregate orthograde apical plug in teeth with wide open apexes: a preliminary study. Oral Surg Oral Med Oral Pathol Oral Radiol Endod. 2009 Apr; 107(4):e52-6.
21. Jacobovitz M, Vianna ME, Pandolfelli VC, Oliveira IR, Rossetto HL, Gomes BP. Root canal filling with cements based on mineral aggregates: an in vitro analysis of bacterial microleakage. Oral Surg Oral Med Oral Pathol Oral Radiol Endod. 2009 Jul;108(1):140-4.

22. Ahlberg KM, Assavanop P, Tay WM. A comparison of the apical dye penetration patterns shown by methylene blue and india ink in root-filled teeth. Int Endod J. 1995 Jan;28(1):30-4.

23. Tanomaru Filho M, Figueiredo FA, Tanomaru JM. Effect of different dye solutions on the evaluation of the sealing ability of Mineral Trioxide Aggregate. Braz Oral Res. 2005 Apr-Jun;19(2):119-22.

24. Lolayekar N, Bhat SS, Hegde S. Sealing ability of ProRoot MTA and MTA-Angelus simulating a onestep apical barrier technique-an in vitro study. J Clin Pediatr Dent. 2009 Summer;33(4):305-10.

25. Komabayashi T, Spangberg LS. Comparative analysis of the particle size and shape of commercially available mineral trioxide aggregates and Portland cement: a study with a flow particle image analyzer. J Endod. 2008 Jan;34(1):94-8.

26. Han L, Okiji T, Okawa S. Morphological and chemical analysis of different precipitates on mineral trioxide aggregate immersed in different fluids. Dent Mater J. Oct 14;29(5):512-7.

27. Bates CF, Carnes DL, del Rio CE. Longitudinal sealing ability of mineral trioxide aggregate as a root-end filling material. J Endod. 1996 Nov;22 (11):575-8.

28. Stefopoulos S, Tsatsas DV, Kerezoudis NP, Eliades G. Comparative in vitro study of the sealing efficiency of white vs grey ProRoot mineral trioxide aggregate formulas as apical barriers. Dent Traumatol. 2008 Apr;24(2):207-13.

\section{CORRESPONDENCIA}

Gilma Esther Romero Romero

Correo electrónico: gerr77@hotmail.com

adiazc1@unicartagena.edu.co 